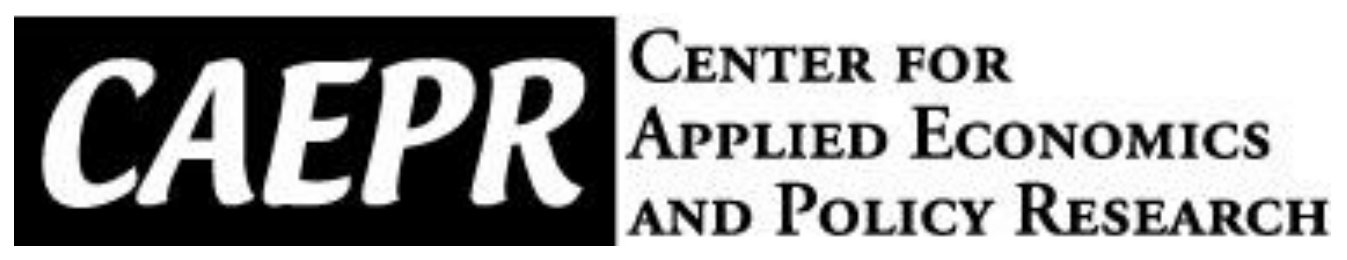

CAEPR Working Paper

\#2012-004

\title{
The Variance Drain and Jensen's Inequality
}

\author{
Robert A. Becker \\ Indiana University
}

March 19, 2012

This paper can be downloaded without charge from the Social Science Research Network electronic library at: http://ssrn.com/abstract=2027471.

The Center for Applied Economics and Policy Research resides in the Department of Economics at Indiana University Bloomington. CAEPR can be found on the Internet at: http://www.indiana.edu/ caepr. CAEPR can be reached via email at caepr@indiana.edu or via phone at 812-855-4050.

(C)2012 by Robert A. Becker. All rights reserved. Short sections of text, not to exceed two paragraphs, may be quoted without explicit permission provided that full credit, including $\odot$ notice, is given to the source. 


\title{
The Variance Drain and Jensen's Inequality*
}

\author{
Robert A. Becker \\ Indiana University
}

March 19, 2012

\begin{abstract}
The well-known approximation of the difference between the arithmetic average and geometric average returns as one-half of the variance of the underlying returns is reexamined using Jensen's Inequality. The "defect" in Jensen's Indequality, is given an exlicit formula in terms of the variance following some ideas put forward by Holder. A new form of the AM-GM Inequality follows and is is applied to financial returns. Both exact, and approximate relations between the arithmetic average, geometric average, and variance of returns are discussed. The mathematical formulation of these relations are free of distributional assumptions governing the underlying returns process.

JEL Classification Numbers: C80, G00, G10
\end{abstract}

\section{Introduction}

That arithmetic average returns for financial assets exceed the corresponding geometric average returns is a well-known consequence of the Arithmetic Mean - Geometric Mean (AM-GM) Inequality. Some authors, such as Cornell ([1], p. 38), observe that the more variable a given asset's return is, the greater the difference between the arithmetic and geometric averages. One way to see how this might be so is to recall a popular approximation:

$$
R_{G} \approx R_{A}-\frac{1}{2} \sigma^{2}
$$

where $R_{A}$ is the arithmetic average return to the asset or portfolio under consideration, $R_{G}$ is its geometric average return, and $\sigma^{2}$ is its variance. ${ }^{1}$ Cornell's

\footnotetext{
*This paper's origins derive from teaching an advanced undergraduate class on financial economics as well as from my reading in the mathematical inequalities literature.

${ }^{1}$ This approximation appears in many texts intended for general readers as well as professionals. Siegel [20] cites this in the second appendix of his first chapter. Dimson, et al ([3], p. 182) report this relation is supported by calculations using their data for market returns to stocks and bonds in a range of countries. More specifically, they ([3], p. 182) comment on the accuracy of this approximation if returns have a distribution that is close to lognormal and note examples where it is fairly accurate in their data sets. Ilmanen ([9], p. 485) comments on how a simple Taylor expansion, to be supplied by the reader, yields (1).
} 
observation can be modeled in terms of this approximation by writing

$$
R_{A}-R_{G} \approx \frac{1}{2} \sigma^{2}
$$

Thus, expressions (1) and (2) suggest that the variance of returns drains the arithmetic average returns to produce the smaller, realized, compound returns over the holding period. ${ }^{2}$ For long holding periods, observed returns for many asset classes appear to satisfy this second form of the approximation with a high degree of accuracy, say an error less than 10 basis points. ${ }^{3}$

Several attempts to derive the approximation formula (1) appear in the finance literature. Markowitz [18] develops several quadratic approximations to discuss long-run returns in terms of means and variances. However, his formulations differ from the ones expressed above. Renshaw [19] attributes the approximation (1) to Lawrence Fisher. There are likely other precursers as well. ${ }^{4}$ Subsequently, Young and Trent [22] and later, Messmore [17], derived versions of this approximation using Taylor polynomials. In the case of Young and Trent's derivation, consideration was also given to higher order approximations recognizing the influence of skewness and/or kurtosis as well as the variance on the magnitude of the spread between the arithmetic and geometric averages. La Grandville [7] includes a severe critique of the approximation formula. He derives an exact form of the relationships between the arithmetic and geometric averages as well as the variance of returns under the additional assumptions that returns are log-normally distributed and compounded continuously.

The purpose of my paper is to show the approximation (2) arises naturally from the AM-GM Inequality. This derivation depends only on realized returns and not on any assumption about the statistical model underlying the returns. In fact, I derive an exact relationship from the AM-GM Inequality that connects the two average returns to the variance of the returns series. A different way of looking at Jensen's Inequality, and how it might be used to prove the AMGM Inequality, does the trick. The arguments follow along lines opened by Holder [8] in his original paper containing his famous inequality. The basic construction, called Holder's defect, is a measure of the distance between the larger and smaller sides in Jensen's Inequality. The exact relationship between average returns and the variance is obtained by applying the natural logarithm function to Jensen's inequality and picking up the role of Holder's defect in the formula. The approximation formula (2) then follows from a first-order Taylor approximation for the natural logarithm function assuming that the arithmetic average returns are "sufficiently small."

\footnotetext{
${ }^{2}$ See Messmore [17] for additional discussion of the variance drain concept.

${ }^{3}$ Morningstar's (2011 Classic Yearbook [13], Table 2.1, p.32) displays arithmetic and geometric average returns as well as the standard deviation of returns for US data for 1926-2010. Both sides of (2) can be computed from that data. The error is the number $R_{A}-R_{G}-\sigma^{2} / 2$. The largest error arises in the small cap stocks portfolio; it is about 71 basis points. The other errors are in the 5-8 basis points range.

${ }^{4}$ The approximation appears as an exercise (p. 45, reference to Exercise 2.3) in Stuart and Ord [10]. Young and Trent ([22], p. 182) reference an earlier text by Yule and Kendall. They go on to note that as of 1969 , there was no published derivation of (1).
} 
The exact relation between the AM, GM and the variance is shown for general conditions when there are a finite number of given positive numbers which may be unequally weighted when forming averages. I follow with a specialization appropriate to the case of financial returns over a long, but finite, holding period where periodic returns are measured over fixed intervals of time, such as one year. The exact relations and the approximation (2) are found for financial applications. Typical uses of (2), such as those found in Cornell [1] and Siegel [20], are for annual returns series observed over several decades. For example, the Morningstar series of returns to large cap stocks, small cap stocks, long term government and corporate bonds, intermediate government bonds, and Treasury bills, [13] referred to below, are collected for the period 1926-2010.

Before going on to outline the paper it is important to point out two small points about approximations such as (1) and (2). In financial applications returns are flow variables measured as percents per unit time. For example, returns in Morningstar's [13] series or the ones in Dimson, et al [3] are reported on annual basis. The first point now follows: neither approximation formula makes sense for the intended financial application unless the factor $1 / 2$ has a dimension. The way in which I derive Holder's defect brings out the way this factor arises in the mathematics.

Second, I assign a precise meaning to the stacked squiggles, or "nearly equal to" symbol, $\approx$. The specific meaning of the expression $(2)$ is that the two sides are equal except for a dimensionless factor close to one that is applied to the righthand side of the relation. ${ }^{5}$ This factor will turn out to be connected to Holder's defect.

Section II goes over Holder's basic result since it is not well-known. Section III supplies the connection to the AM-GM Inequality. Section IV shows the link between the approximation formula (1) and the exact relationship expressed in (2). Concluding comments complete the paper.

\section{Jensen's Inequality, Holder's Defect and the Variance}

Let $f$ be a twice continuously differentiable real-valued function defined on the compact interval $[a, b]$ with $a<b$. Assume that $f$ is a convex function: that is, $f^{\prime \prime}(x) \geq 0$ for each $x$. Recall that if $f^{\prime \prime}(x)>0$ for each $x$, then $f$ is strictly convex.

Suppose that $X$ is a random variable defined on a probability space that takes one of the values $\left\{x_{1}, x_{2} \ldots, x_{N}\right\} \subseteq[a, b], N$ a natural number, and $\operatorname{Pr}\{X=$ $\left.x_{k}\right\}=p_{k}>0$, with $\sum_{k=1}^{N} p_{k}=1$. Define the expected value of $X$ by the formula

$$
E(X)=\sum_{k=1}^{N} p_{k} x_{k} .
$$

\footnotetext{
${ }^{5}$ I follow the usage in Mahajan ([16], p. 6).
} 
The alternative notation $x_{A}=E(X)$ is also used in several equations to save space. Obviously $x_{A} \in[a, b]$. Denote the expected value of the function $f$ by:

$$
E(f(X))=\sum_{k=1}^{N} p_{k} f\left(x_{k}\right) .
$$

The variance of $X$ is expressed by the formula

$$
\operatorname{Var}(X)=\sum_{k=1}^{N} p_{k}\left(x_{k}-x_{A}\right)^{2} .
$$

The alternative notation $\sigma^{2}=\operatorname{Var}(X)$ is used to save space in some equations.

Jensen's Inequality states that if $f$ is a finite, real-valued convex function defined on $[a, b]$, then

$$
E(f(X)) \geq f(E(X)) .
$$

If $f$ is strictly convex, then there is equality in (6) if and only if $\operatorname{Var}(X)=0$, that is, $x_{k}=x_{A}$ for each $k$.

The notation in (6) is based on a probabilistic interpretation of Jensen's Inequality. However, I do not insist on this interpretation. The formal advantage of doing so is in the statement of the Theorem 1 and its applications in which the variability of the numbers $\left\{x_{1}, \ldots, x_{N}\right\}$ is of primary concern.

It is obvious that inequality (6) can be recast as an equality in the case where $f$ is strictly convex and $\operatorname{Var}(X)>0$ by finding an appropriate positive number, $\Delta$, depending on $X$, such that

$$
E(f(X))=f(E(X))+\Delta .
$$

The problem addressed below is to find a formula expressing the defect, $\Delta$, in terms of the given numbers $\left\{x_{1}, x_{2} \ldots, x_{N}\right\}$ and their corresponding probabilities. There is no loss of generality in assuming that $a \leq x_{1} \leq x_{2} \leq \cdots \leq x_{N} \leq b$ obtains.

Holder's [8] defect appears in his famous paper where he first established the inequality bearing his name (see [21] for additional discussion). Using the probabilistic notation, Holder's Defect formula is expressed in equation (9) below. ${ }^{6}$

Theorem 1 If $f:[a, b] \rightarrow \mathbb{R}$ is twice continuously differentiable and there are finite bounds $m$, and $M$ such that

$$
0 \leq m \leq f^{\prime \prime}(x) \leq M \text { for each } x \in[a, b],
$$

then for any real-values $a \leq x_{1} \leq x_{2} \leq \cdots \leq x_{N} \leq b$ and any nonnegative real numbers $p_{k}, k=1,2, \ldots, N$ with $\sum_{k=1}^{N} p_{k}=1$, there exists a real value $\mu \in[m, M]$ for which the formula (9) obtains:

$$
E(f(X))-f(E(X))=\frac{1}{2} \mu \operatorname{Var}(X) .
$$

\footnotetext{
${ }^{6}$ Steele ([21], p.94) gives an equivalent statement to mine. See Remark 2 for his version. Cerone and Dragomir ([2], pp. 48-50), based on Dragomir [4], report bounds for Jensen's Inequality in terms of the second derivative. Dragomir's work appears to be independent of Holder's original statement.
} 
Holder's Defect says that $\Delta$ is proportional to the random variable $X$ 's variance. The number $\mu \operatorname{Var}(X) / 2$ added to the right-hand side is the Jensen's inequality "correction" in estimating the expected value of the function $f$ of the random variable $X$ by the function $f$ evaluated at the random variable's mean. An elegant proof of this formula can be found in Steele ([21], pp. 94-96), who, in turn, follows Holder's reasoning. I propose a different proof below based on Taylor's Theorem. It is likely that many authors have come across part of this argument (the use of Taylor's Theorem to prove Jensen's Inequality for twice continuously differentiable functions) but not taken the additional step to show how Holder's formula emerges. Indeed, Hardy, Littlewood, and Polya ([6], p.151) is one such example. ${ }^{7}$

Proof. Apply Taylor's Theorem with the Lagrange form of the remainder to $f$ at the point $x_{A}$ in its domain to obtain for each $x_{k} \in[a, b], k=1,2, \ldots, N$ :

$$
f\left(x_{k}\right)=f\left(x_{A}\right)+f^{\prime}\left(x_{A}\right)\left(x_{k}-\bar{x}\right)+\frac{1}{2} f^{\prime \prime}\left(c_{k}\right)\left(x_{k}-x_{A}\right)^{2},
$$

where $c_{k}$ is a real number between $x_{k}$ and $x_{A}$. Multiply the above equation by $p_{k}$ and sum to obtain after simplifying:

$$
\sum_{k=1}^{N} p_{k} f\left(x_{k}\right)=f\left(x_{A}\right)+\frac{1}{2} \sum_{k=1}^{N} p_{k}\left(x_{k}-x_{A}\right)^{2} f^{\prime \prime}\left(c_{k}\right) .
$$

That is,

$$
E(f(X))-f(E(X))=\frac{1}{2} \sum_{k=1}^{N} p_{k}\left(x_{k}-x_{A}\right)^{2} f^{\prime \prime}\left(c_{k}\right) .
$$

This expresses Holder's defect in terms of the generally unknown values $c_{k}$. Note that since $f^{\prime \prime} \geq 0$, equation (12) demonstrates Jensen's Inequality for the twice continuously differentiable convex function $f$. To prove (9) use the assumed bounds on $f^{\prime \prime}$ to observe that ${ }^{8}$

$$
\frac{m}{2} \operatorname{Var}(X) \leq \frac{1}{2} \sum_{k=1}^{N} p_{k}\left(x_{k}-x_{A}\right)^{2} f^{\prime \prime}\left(c_{k}\right) \leq \frac{M}{2} \operatorname{Var}(X) .
$$

The Intermediate Value Theorem applied to the function

$$
\sigma(t):=\frac{t}{2} \operatorname{Var}(X), t \in[m, M]
$$

\footnotetext{
${ }^{7}$ To be fair, Hardy, Littlewood and Polya use Taylor's Theorem to demonstrate Jensen's Inequality for integrals instead of the finite sums that concern me in this note. Economists have also come across this use of Taylor's Theorem for proving Jensen's Inequality. For example, see Hirshleifer and Riley ([12] , p.32). They do not make the additional step to derive Holder's defect formula.

${ }^{8}$ Note that $f^{\prime \prime}$ continuous on $[a, b]$ implies $m$ and $M$ are each achieved at some point in the interval $[a, b]$. That is, there are points $\alpha$ and $\beta$ in $[a, b]$ such that $m=f^{\prime \prime}(\alpha) \leq f^{\prime \prime}\left(c_{k}\right) \leq$ $f^{\prime \prime}(\beta)=M$ for each $c_{k}$. Cerone and Dragomir [2] as well as by Dragomir [4] emphasize these variance bounds in their presentation of Jensen's Inequality and their refinement of the AMGM inequality. They do not take the step of identifying the middle term of the inequality with a scaler multiple of the variance, as is done below.
} 
implies there is a $\mu \in[m, M]$ such that

$$
\frac{\mu}{2} \sum_{k=1}^{N} p_{k}\left(x_{k}-x_{A}\right)^{2}=\frac{1}{2} \sum_{k=1}^{N} p_{k}\left(x_{k}-x_{A}\right)^{2} f^{\prime \prime}\left(c_{k}\right) .
$$

Substituting the LHS of (14) into the RHS of (12) proves the theorem.

The factor $1 / 2$ arises in the proof by way of the remainder term in the firstorder Taylor approximation. This factor has a dimension in applications such as to financial data. ${ }^{9}$

Notice that for strictly convex functions that a positive variance implies Jensen's Inequality is strict. More important, the defect, or correction, necessary to restore equality in Jensen's Inequality depends only on the variance of $X$ and not directly on any higher central moment. The factor $\mu$ absorbs the impact of all higher order moments associated with $X$.

Steele [21] writes (9) in an equivalent form of Remark 2 and provides Examples 3 and 4 as well.

Remark 2 Holder's Defect (9) has the equivalent expression: there is a $\mu \in$ $[m, M]$ such that

$$
E(f(X))-f(E(X))=\frac{1}{4} \mu \sum \sum p_{j} p_{k}\left(x_{j}-x_{k}\right)^{2} .
$$

Proof. An elementary computation shows $\sum_{j=1}^{N} \sum_{k=1}^{N} p_{j} p_{k}\left(x_{j}-x_{k}\right)^{2}=2 \operatorname{Var}(X)$.

Example 3 If $f^{\prime \prime}(x)=0$ for each $x$, then $m=M=0$ and $\Delta=0$.

Example 4 Let $f(x)=x^{2}$; then $\operatorname{Var}(X)=E\left(X^{2}\right)-(E(X))^{2}$ and $\mu=2$, which is a constant independent of the particular values $x_{k}$, so that we have the quadratic identity

$$
E(f(X))-f(E(X))=\operatorname{Var}(X) .
$$

A more interesting example is based on the convexity of $-\ln x$. This is the subject of the next section.

\section{Jensen's Inequality, Holder's Defect, and the AM-GM Inequality}

The general form of the Arithmetic Mean - Geometric Mean Inequality for positive numbers $x_{k}$ and $p_{k}$, with $\sum_{k=1}^{N} p_{k}=1$, is

$$
\sum_{k=1}^{N} p_{k} x_{k} \geq \prod_{k=1}^{N} x_{k}^{p_{k}}, \text { with equality if and only if } x_{k}=x_{A} \text { for each } k,
$$

\footnotetext{
${ }^{9}$ Dimensions are distinct from units. The units for returns depend on the frequencey of the underlying data. For example, annual returns data versus monthly returns. The factor $1 / 2$ must have appropriate unit time per percentage units that match the measurement of the arithmetic and geometric averages.
} 
where $\sum_{k=1}^{N} p_{k} x_{k}=x_{A}$ denotes the arithmetic mean, or average, of the numbers $\left\{x_{1}, x_{2} \ldots, x_{N}\right\}$. Let $x_{G}=\prod_{k=1}^{N} x_{k}^{p_{k}}$ denote the corresponding geometric mean, or average, of the numbers $\left\{x_{1}, x_{2} \ldots, x_{N}\right\}$. This notation does not emphasize the probabalistic interpretation given in the previous section. The special case where $p_{k}=1 / N$ for each $k$ is of interest, particularly in the financial market context.

The AM-GM Inequality has many demonstrations. One way to prove it is by applying Jensen's Inequality to the strictly convex function $-\ln x$ on the interval $[a, b]$ with $0<a<b<+\infty$. Holder's defect formula applied to $-\ln x$ yields the following form of the AM-GM Inequality under the same assumptions as Theorem 1:

Proposition 5 There is a positive number $\mu$ (depending on $X$ ) such that

$$
\left(\sum_{k=1}^{N} p_{k} x_{k}\right) \exp \left(-\frac{\mu}{2} \sum_{k=1}^{N} p_{k}\left(x_{k}-x_{A}\right)^{2}\right)=\prod_{k=1}^{N} x_{k}^{p_{k}} .
$$

Moreover,

$$
\left(\sum_{k=1}^{N} p_{k} x_{k}\right)=\prod_{k=1}^{N} x_{k}^{p_{k}} \text { iff } \sum_{k=1}^{N} p_{k}\left(x_{k}-x_{A}\right)^{2}=0 .
$$

Notice that the assumption $0<a \leq x_{1} \leq x_{2} \leq \cdots \leq x_{N} \leq b<+\infty$ implies that $\mu \in\left[x_{N}^{-1}, x_{1}^{-1}\right]$.

Proof. Equation (9) for $f(x)=-\ln x$ yields, after rearranging,

$$
\ln \bar{x}_{A}-\frac{\mu}{2} \sum_{k=1}^{N} p_{k}\left(x_{k}-x_{A}\right)^{2}=\sum_{k=1}^{N} p_{k} \ln x_{k} .
$$

Exponentiate both sides and simplify to obtain (18). Note,

$$
\exp \left(-\frac{\mu}{2} \sum_{k=1}^{N} p_{k}\left(x_{k}-x_{A}\right)^{2}\right) \leq 1,
$$

with strict inequality whenever $\sum_{k=1}^{N} p_{k}\left(x_{k}-x_{A}\right)^{2}>0$.

The Arithmetic Mean - Geometric Mean Inequality (17) follows from the Proposition since (20) holds. The additive term in (19) corresponding to Holder's Defect $(\Delta=\mu \operatorname{Var}(X) / 2)$ from Jensen's Inequality turns into a multiplicative term upon exponentiation.

This way of looking at the AM-GM Inequality highlights the role of the variance of $X$ in determining whether or not there is an equality, or inequality, between the arithmetic and geometric means of the positive numbers $\left\{x_{1}, x_{2} \ldots, x_{N}\right\}$. Returning to the probabilistic setting, Proposition 5 says no higher order central 
moment of the random variable $X$ matters for settling the question of equality, or inequality between $x_{A}$ and $x_{G}$. Of course, higher order moments can influence the magnitude of the factor $\mu{ }^{10}$

Equation (19) can be rewritten as

$$
\ln x_{A}=\ln x_{G}+\frac{\mu \sigma^{2}}{2}
$$

where $\sigma^{2}=\sum_{k=1}^{N} p_{k}\left(x_{k}-x_{A}\right)^{2}$ is now used to denote the variance of the numbers $\left\{x_{1}, x_{2} \ldots, x_{N}\right\}$. This equation represents the exact mathematical relationship between the arithmetic and geometric means concepts and the variance of $X$. Of course, this equation can be rewritten as

$$
\ln \left(\frac{x_{A}}{x_{G}}\right)=\frac{\mu \sigma^{2}}{2},
$$

instead. Moreover, it is trivial to solve for the value of $\mu$ in terms of $x_{A}, x_{G}$ and $\sigma^{2}$. The development of approximate versions of equation (21) for market returns data is proposed in the next section. The main idea behind the approximation formulas is that for $x$ sufficiently close to 1 , the first-order approximation $\ln x=x-1$ can be applied to $(21)$ to yield $x_{A}-x_{G}$ is nearly equal to $\mu \sigma^{2} / 2$. The market returns application puts $x_{t}=1+r_{t}$ where $r_{t}$ is the observed net return in period $t$.

\section{Market Returns, the AM-GM Inequality, and Approximations}

Stock and bond market average annual returns are usually reported in two ways. Once as the arithmetic average annual returns measured as a percentage per year, denoted $R_{A}$, and again as the geometric (or, compound) average annual returns, likewise measured as a percentage per year and denoted by $R_{G} .{ }^{11}$ The data underlying these averages consists of annual net returns, $r_{t}$, where $r_{t}$ is the annual return on the underlying portfolio of stocks and/or bonds in question in year $t$ and is measured as a percentage per year. Annual et returns are measured, for example, as the profit in dollars per dollar (expressed as a percentage) invested per year.

The numbers $r_{t}$ for $t=1,2, \ldots, T$ are usually thought of as the outcomes of an underlying random returns process governing market returns in either the stock or bond market, depending on the application in mind. Put differently, these returns are observations in a sample over a holding period based on the

\footnotetext{
${ }^{10}$ Young and Trent [22] arrive at an exponential function of a fourth order Taylor polynomial as the basis for their approximation formulas. Their use of the exponential formulation is close to the approach taken here, except the variable in their polynomial is expressed in terms of deviations from the mean in order to bring the variance, skewness, and kurtosis together.

${ }^{11}$ See [5] and [3] for two such examples of reporting annual returns for US and foreign markets. Reference [13] includes annual and some higher frequency returns data.
} 
historical performance of the asset class in question. Other applications are possible for reported inflation and exchange rates as well as real versus nominal returns. The numbers $1+R_{A}, 1+R_{G}$, and $1+r_{t}$ are the gross returns per year. Limited liability implies that $1+r_{t} \geq 0$ for each year data is reported. The typical financial application of the approximation formulas is usually reserved for large divesified portfolios of stocks and/or bonds where $1+r_{t}>0$ is actually observed in every period under study.

Fix the finite sequence of numbers $\left\{r_{1}, r_{2}, \ldots, r_{T}\right\}$. The average gross annual return is defined by

$$
1+R_{A}=\frac{1}{T} \sum_{t=1}^{T}\left(1+r_{t}\right) .
$$

The compound average gross annual return is defined by the formula

$$
1+R_{G}=\left(\prod_{t=1}^{T}\left(1+r_{t}\right)\right)^{1 / T},
$$

where $\Pi_{t=1}^{T} \xi_{t}$ denotes the product of the given factors $\xi_{t}$. The compound average net annual return is just found by rewriting the previous equation as

$$
R_{G}=\left(\prod_{t=1}^{T}\left(1+r_{t}\right)\right)^{1 / T}-1
$$

$R_{G}$ is the number reported as the geometric mean in the market returns data. Likewise, $R_{A}$ is the arithmetic mean recorded in the data based on realized net returns.

The classical Arithmetic Mean-Geometric Mean (AM-GM) Inequality implies

$$
1+R_{A} \geq 1+R_{G}
$$

with equality if and only if there is a number $\bar{r}$ such that $r_{t}=\bar{r}$ for each $t$. Obviously, this inequality is equivalent to $R_{A} \geq R_{G}$ with equality if and only if $r_{t}=\bar{r}$ for each $t$. The case of strict inequality occurs when the values of $r_{t}$ are unequal - their variance, $\sigma^{2}$, is positive. The formal result of Proposition 5 is that there is a positive number $\mu$, depending on the finite sequence $\left\{r_{1}, r_{2}, \ldots, r_{T}\right\}$ such that

$$
\left(1+R_{A}\right) \exp \left(-\frac{\mu}{2} \sigma^{2}\right)=\left(1+R_{G}\right)
$$

There is equality between $R_{A}$ and $R_{G}$ if and only if $\sigma^{2}$ is zero. Evidently $p_{k}=1 / N$ for each $k$ is assumed in this application. Taking natural logarithms of both sides of the above equation to obtain

$$
\ln \left(1+R_{A}\right)+\left(-\frac{\mu}{2} \sigma^{2}\right)=\ln \left(1+R_{G}\right)
$$

Only the natural logarithms of the returns stands in the way of verifying (2). Now suppose that $R_{A}$ is sufficiently small that the approximation $\ln \left(1+R_{A}\right) \simeq$ 
$R_{A}$ holds. ${ }^{12}$ Then $R_{A} \geq R_{G}$ implies that the same error, or smaller, would hold and justify taking the approximation $\ln \left(1+R_{G}\right) \simeq R_{G}$. Then the approximate formula derived from (28) holds:

$$
R_{A}+\left(-\frac{\mu}{2} \sigma^{2}\right) \simeq R_{G}
$$

If equality replaces the symbol $\simeq$, then, after rearrangement, $(29)$ is the same as (2) except for the parameter $\mu$. IF $\mu \approx 1$, then (2) obtains as an approximate relation between the two average return measures and the variance in returns. In fact, for the large cap, long-term corporate, and government bond series compiled by Morningstar [13], the exact value of $\mu$ found from solving (28) is about .85. Their small cap series $\mu$ is about .75 and the intermediate governments value is about .58. On the other hand, the T-bills value is 2 , which seems strange except that one-half the variance is about 5 basis points, almost an order-of-magnitude smaller than the corresponding figure for the long bonds. In the T-bill case $R_{A}$ differs from $R_{G}$ by about 10 basis points, which is also the value of the variance of the returns with its dimensions modified to make it comparable in units to the average returns units. The approximation (2) is off by about 5 basis points. Thus, it seems reasonable, even if $\mu$ is not always very close to 1 , at least for long holding periods, that approximations carried out in (29) are about as accurate as the ones in (2), and to write:

$$
R_{A}-R_{G} \approx-\frac{1}{2} \sigma^{2}
$$

which is the approximation formula promised in the introduction.

\section{Conclusion}

The derivation of the approximate returns formulas from Jensen's Inequality necessarily follows from assumptions that go along with the use of first-order Taylor approximations. However, the accuracy of the approximating formulas (29) and (2) are empirical matters that depend on the chosen application. Here, long-holding period returns are used to assess the potential errors from using one approximation, or the other, compared to calculating the exact relationship. The errors computed for the basic Morningstar series's applied to (29), compared to the exact relation (28), rearranged as:

$$
\ln \left(1+R_{A}\right)-\ln \left(1+R_{G}\right)=\frac{\mu}{2} \sigma^{2},
$$

\footnotetext{
${ }^{12}$ The notation $\simeq$ is used to convey this relation is one of approximation and not equality, even after multiplying the right-hand side by some constant. If the positive average return $R_{A}$ is smaller than about $15 \%$, then the approximation $\ln \left(1+R_{A}\right) \simeq R_{A}$ overestimates $\ln \left(1+R_{A}\right)$ by no more than $1 \%$. For arithmetical average returns for the large cap, various long-term bond series, and T-Bills produced by Morningstar the largest error from this approximation is closer to 70 basis points. The Morningstar small cap series in [13] measures an arithmetic average return of almost $17 \%$, which falls just outside the range of the first-order approximation for $\ln \left(1+R_{A}\right)$ used here. Of course, that series has the largest variance of the basic ones reported in that source.
} 
are on the order of 2 basis points for long bonds, about 20 basis points for the large cap series, and about 60 basis points for the small cap series. Intermediate bonds and T-bills have errors that are basically zero given the number of significant digits in the underlying data. Similar orders of magnitude occur for the errors arising from the use of (2). In short, the approximation (2) is reasonably robust even after adjusting it to account for the contribution of Holder's defect to the difference between the arithmetic and geometric average returns. 


\section{References}

[1] Bradford Cornell, The Equity Risk Premium: The Long-Run Future of the Stock Market, John Wiley \& Sons, New York, 1999.

[2] Pietro Cerone and Sever S. Dragomir, Mathematical Inequalities: A Perspective, CRC Press, Boca Raton, 2011.

[3] Elroy Dimson, Paul Marsh, and Mike Staunton, Triumph of the Optimists: 101 Years of Global Returns, Princeton University Press, Princeton, 2002.

[4] Sever S. Dragomir, "Some Inequalities for $(m, M)$-Convex Mappings and Applications for the Csiszár $\Phi$-Divergence in Information Theory," Math. J. Ibaraki University, 2001 (33), 35-50.

[5] William N. Goetzmann and Roger G. Ibbotson, The Equity Risk Premium: Essays and Explorations, Oxford University Press, 2006.

[6] G.H. Hardy, J.E. Littlewood, and G. Polya, Inequalities, Second Edition, Cambridge University Press, 1951 [1967 printing].

[7] Olivier de La Grandville, "The Long-Term Expected Rate of Return: Setting It Right," Financial Analysts Journal, November-December 1998 (54), $75-80$.

[8] Otto Hölder, "Ueber einen Mittelwerthabsatz," Nachrichten von der Königl. Gesellschaft der Wissenschaften und der Georg-AugustsUniversität zu Göttiingen, Band 1889 (2), 38-47.

[9] Antii Ilmanen, Expected Returns, John Wiley \& Sons, New York, 2011.

[10] Alan Stuart and Keith Ord, Kendall's Advanced Theory of Statistics, Sixth Edition, Volume 1: Distribution Theory, Oxford University Press, New York, 1994.

[11] John Y. Campbell, Andrew W. Lo, and A. Craig MacKinley, The Econometrics of Financial Markets, Princeton University Press, Princeton, 1997.

[12] Jack Hirshleifer and John G. Riley, The Analytics of Uncertainty and Information, Cambridge University Press, 1992.

[13] Ibbotson SBBI 2100 Classic Yearbook: Market Results for Stocks, Bonds, Bills, and Inflation: 1926-2010, Morningstar, Inc., Chicago, 2011.

[14] Eric Jacquier, Alex Kane, and Alan J. Marcus, "Geometric or Arithmetic Mean: A Reconsideration," Financial Analysts Journal, NovemberDecember 2003 (59), 46-53.

[15] H. Levy and H.M. Markowitz, "Approximating Expected Utility by a Function of Mean and Variance," The American Economic Review, June 1979 (69), 308-317. 
[16] Sanjoy Mahajan, Street-Fighting Mathematics: The Art of Educated Guessing and Opportunistic Problem Solving, The MIT Press, Cambridge, 2010.

[17] Tom Messmore, "Variance Drain," The Journal of Portfolio Management, Summer 1995 (21), 104-110.

[18] Harry M. Markowitz, Portfolio Selection: Efficient Diversification of Investments, Yale University Press, New Haven, 1959.

[19] Edward F. Renshaw, "Portfolio Balance Models in Perspective: Some Generalizations that Can be Derived from the Two-Asset Case," The Journal of Financial and Quantitative Analysis, 1967 (2), 123-149.

[20] Jeremy Siegel, Stocks for the Long-Run, Fourth Edition, McGraw-Hill, 2008.

[21] J. Michael Steele, The Cauchy-Schwartz Master Class: An Introduction to the Art of Mathematical Inequalities, Cambridge University Press and Mathematical Association of America, 2004.

[22] William E. Young and Robert H. Trent, "Geometric Mean Approximations of Individual Security Returns and Portfolio Performance," The Journal of Financial and Quantitative Analysis, June 1969 (4), 179-199. 